\title{
The System of High Current Full Fiber Detection
}

\author{
Chengjun Guo ${ }^{1}$, Ning Pei ${ }^{2, *}$, Jixiang $\mathrm{Lu}^{1}$ and Jin Wang ${ }^{1}$ \\ ${ }^{1}$ College of science and technology Ningbo University, Ningbo Zhejiang \\ ${ }^{2}$ Inner Mongolia Institute of Metal Materials, Ningbo Zhejiang \\ ${ }^{*}$ Corresponding author
}

\begin{abstract}
Current and voltage measurements play an important role in the power industry and provide necessary information for power systems to measure, control, and relay protection. At present in the national $110 \mathrm{KV}, 110 \mathrm{KV}, 330 \mathrm{KV}$, $750 \mathrm{KV}$ power grid has been fully tested by optical current transformer, at the same time as the solar and wind power mature gradually, the establishment of the smart grid is imperative, also need the original optical current transformer as a test. It is expected that all optical current transformers will gradually replace the electromagnetic type transformers for smart grid and high voltage grid in the future. Therefore, the demand of market will be huge.
\end{abstract}

Keywords-full fiber; formatting; current; sensor

\section{INTRODUCTION}

The full optical fiber current sensor is a branch of optical fiber sensing, resistance to electromagnetic interference, high temperature resistant, resistant to chemical corrosion, high security., can be used in power plants, substation, transmission line voltage, current and other parameters monitoring. The fullfiber current sensor of the ring cavity structure and the whole fiber current sensor of the multi-channel ring cavity structure are discussed in detail. In order to improve the sensitivity of optical fiber current sensor, a full - fiber current sensor with ring cavity structure is proposed. The structure of the sensor take the light pulse cycle along the same direction through the optical fiber sensing head, made by the magnetic field caused by Faraday rotation Angle is gradually increased, to a large extent make up for the defect of the ordinary single mode optical fiber verdet constant. From Faraday magneto optical effect, by means of Jones vector, using the theory of optical fiber sensor transfer matrix to establish the structure analysis model, deduced the structure of the full optical fiber current sensor polarization degree, the mathematical expressions of sensitivity, the necessary of numerical calculation and experimental verification, the results show that the ring cavity of the polarization degree of the full optical fiber current sensor and current between certain linearity, sensitivity and current with the cycle of light pulse in a ring cavity number $\mathrm{K}$ wave rise, reasonably choose $\mathrm{K}$ value is very important to control the sensitivity of the whole system. Based on the current demand of the large scale and multi-channel measurement current in the current power grid, this paper presents a full-fiber current sensor for the multi-channel ring cavity structure. The same condition, compared with single channel current sensor has the advantages of simultaneously measuring wire more current than the parallel structure of optical fiber current sensor has higher energy utilization, achieve the goal of synergistic authors. Experiment set the dual tandem ring cavity structure optical fiber current sensor, the results show that the two polarization of light signals in optical fiber sensing head will change with the current in the conductor, and the change of the degree of polarization and has good linearity and the question of current increases with increasing $\mathrm{K}$, tandem structure fully verified the feasibility of the full optical fiber current sensor. In addition, in view of the series-parallel structure of two kinds of light energy utilization of optical fiber current sensor formula deduction and comparative analysis, the result shows that tandem structure has unique advantages in terms of light energy utilization.

\section{EXPERIMENTAL}

The basic theoretical basis of the all-optical fiber current sensor is the Faraday magnetic and ampere loop theorem. Faraday magneto-optic effect is: when a current flows through a conductor produces electromagnetic fields, the polarized light along the direction parallel to the magnetic field through the sensing optical fiber, the polarization of the linear polarized angle of deflection. The instantaneous current value can be calculated by measuring the variation of the polarization angle. Ampere loop theorem is around a conductor measurement fiber only to the current inside the ring, ring of any magnetic field on magnetic field sensing optical fiber ring closed-loop vector integral is zero, which does not produce interference measurement. Based on Faraday magneto-optic effect, by the method of double beam interference with light path Sagnac Faraday magneto-optic effect of magnetic field measurement, interferometry is used to measure the method of optical path difference of polarized light, finally calculate the instantaneous current value. Its composition principle is shown in figure 1:

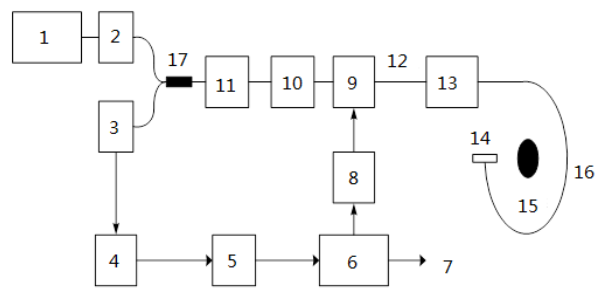

1. light source and its drive, 2. light source, 3.PIN detector, 4.amplifier circuit, 5., A/D 6. information processing system, 7. signal output 8. D/A 9.modulator 10.detector 11.polarizer 12.polarization-maintaining optical fiber 13.a quarter wave plate 14 .mirror 15 . the supply wire of power 16 .multi-loop measurement fiber 17.fiber optic coupler

FIGURE I. FULL FIBER CURRENT SENSOR STRUCTURE 
It can be seen from the above figure that the all-fiber current sensor consists of three parts: the head part, the launch part, the detection and the information processing part. The head part mainly includes: mirror, multi-loop measurement fiber, a quarter wave plate, polarization-maintaining optical fiber, etc. The launching part mainly includes: light source and its drive, fiber optic coupler, polarizer, detector, modulator, etc. The receiving part mainly includes: PIN detector, amplifier circuit, D/A, A/D, information processing system, etc.

Light source the light through the optical fiber coupler, a partial, partial detector is divided into two after the road into a line of polarized light, a modulator junior slant cancelling a modal fiber, after a quarter wave plate into circularly polarized light (two beams of light spin to the same), along the opposite direction into the measuring optical fiber, more because of the existence of Faraday magneto-optic effect two circularly polarized light beam of polarization plane rotation occurs, when light reflector returns, after a quarter wave plate interference occurs, by measuring the interference light intensity at a certain moment, find out the phase difference, and then get the corresponding current size. When there is no current in the current conductor, the two beams of light are uniformly distributed at the same time as the circular polarization in the optical fiber, and the speed of light is equal to $\mathrm{V}_{0}$. No phase difference is possible, no interference occurs. When the current in the conductor is energized, according to the ampere loop theorem shows that the magnetic field generated around a conductor, the Faraday magneto-optic effect propagation along the direction of the magnetic field of circularly polarized light changes have taken place in the speed of light, the speed of the circular polarization and circularly polarized light direction, the direction of light propagation. Because of the two beams of light in optical fiber transmission in the opposite direction, circularly polarized light of light speed, one is $\mathrm{V}_{1}, \mathrm{~V}_{2}$, so will produce phase difference to produce interference, the principle of the interfere and optical fiber gyro Sagnac effect on the principle which is similar.

(1) laser and its driver: due to the use of interferometry, the high precision of the laser light source is required, and the constant laser power output of high stability is required. A semiconductor laser with a wavelength of $800 \mathrm{~nm}$ can be selected and the spectrum width is less than $5 \mathrm{~nm}$. To ensure its high stability, especially temperature change on the influence of its performance, need to design the drive circuit, drive mainly includes automatic power adjustment, automatic temperature adjustment, software startup and other auxiliary circuit, etc.

(2) phase modulator: sensor response and phase difference is proportional to the cosine function, due to the phase difference is very small, usually measured in exchange, the phase difference of two-way change, so the interferometer sensitivity is low, the solution is to give the interferometer is introduced into a non reciprocal bias phase, in order to improve the measurement accuracy, therefore, including phase modulator in the system structure. In addition, due to the nature of the cosine function, under the condition of low current, low detection sensitivity, therefore, must to detect signal plus a phase deviation, make the output signal is near zero gain maximum sensitivity. Using a variety of modulation signal drives, optical path is proportional to the modulation signal instantaneous value, because of polarization-maintaining fiber and sensing optical fiber delay action of waves, two beams of light through the modulator at different times, different optical path, to achieve the phase offset. According to different modulation signals, the corresponding signal processing algorithm is used to demodulate and get the phase difference. Modulation mode can be divided into two methods of active and passive modulation, passive modulation method is mainly used in the field of optical fiber gyro mature technology, but where is the use of square wave modulation, the frequency of the square wave contains composition is complicated, the rising and falling and offset the volatility will affect the measurement results, the requirement to the response speed is very low, fiber optic gyroscope can be obtained after a large number of sampling points such as average algorithm to eliminate the influence of the above factors, but for optical fiber current transformer, protective current transformer, in particular, have higher demand to the response speed, so the square wave modulation is not applicable. Active modulation method, can use piezoelectric ceramics (PZT) phase modulator, under the modulation signal drive, wrapped around the PZT tube on fiber length change, realize the optical phase modulation, this section can be used in a mature technology.

(3) optimize the light path design to eliminate the influence of temperature and vibration on the measurement results

Sag Nike interferometry fiber optic current transformer has not been widely engineering application, one of its important reason is the transformer for the sensitivity of the environmental vibration and disturbance of environment temperature drift. With similar sag Nike interferometer light path structure, when the light through the optical fiber ring cavity interference occurs, the vibration of the environment or the shaking will lead to circular cavity with instantaneous angular velocity, angular velocity will cause the sag Nike interference effect, sag of Nike interference phase difference with the Faraday effect of phase coupling together cannot be separated, resulting in the error of current measurement. The more severe the vibration of the environment, the greater of the error. Optical fiber coil, on the other hand, the temperature disturbance will cause additional phase shift, and thus generate offset error of current transformer, unless with good thermal insulation structure or design, or be able to eliminate the error. At home and abroad in order to solve the problem, many scholars and researchers have put forward and verified for sag Nike interferometry fiber optic current transformer improvements, such as silica optical fiber using annealing coil, torsion fiber, elliptical birefringence fiber, mirror reflection optical fiber current transformer, symmetric optical fiber current transformer, etc. However, the above schemes still have too complicated structure, expensive cost, strict material and process requirements. The optical path optimization design is carried out to improve the traditional optical path structure. Add a mirror at the end of the coil sensing fiber. Rotating 90o at the coupling angle of a $1 / 4$ wave plate in the input port of the sensor coil. Based on the Jones matrix theory and the experiment results show that compared with the traditional optical fiber current transformer, a new sag Nike interferometry fiber optic current transformer eliminates the sensitivity to the 
environment vibration and disturbance of environment temperature drift.

(4) simulated feedback control scheme: the digital closedloop control system can not solve the zero drift and can hardly reach the measurement accuracy of 0.2s-class. When the environment changes, such as temperature will affect the accuracy of measurement, especially for reading, such as the impact of the largest, 0.0001 per ten degrees of change will affect the readings, appear the phenomenon of zero drift and unstable, can be used in a closed loop feedback control technology, using analog closed-loop control technology here. Although, digital feedback control technology has many advantages, but in the entire system with $\mathrm{A} / \mathrm{D}, \mathrm{D} / \mathrm{A}$, FPGA, DSP and other digital devices, can introduce additional control and delay error, the cause in the process of range measurement error accumulation is too big, is the main cause of instability and zero drift.

(5) miniaturization design: get rid of disadvantages of conventional CT volume big, full optical fiber current sensor can be small, according to its composition characteristics, optimize the system component, will be connected to the host part USES optical fiber sensing probe, the host can be placed in the appropriate position, display parts placed on the ground, or by transferring to the monitoring room. The sensor head is mainly multi-loop optical fiber and its fixed mechanism; Host part in addition to the optics parts, mainly electrical processing part, signal processing using large scale integrated chip of FPGA and DSP completed a combination of high speed information processing, signal processing miniaturization.

(6) signal processing: light detector will contain a light signal into electrical signal to measure information, because the signal is weak, so the first amplifier, then filtering, keep only in the sensor output harmonic component at a time. Then highspeed sampling is carried out to complete the module conversion. Output DSP digital signal except with the display and control equipment interface, according to the power system specification requirements, should also be back to the original on the power bus current waveform, so the system need to D/A converter and low pass filter. The system USES a FPGA chip to generate various control timing, phase modulator carrier and other parts of the control circuit.

As a result of the optical fiber Faraday magneto-optic effect is weak, interferometer output signal is very small, and therefore have higher requirements on characteristics of photoelectric detector, can be selected according to specific requirements PIN detector, the characteristic is high responsively, fast response and low noise, stable and reliable.

To obtain the information of the measured current, one and second harmonic components of the output light intensity signal are required. Therefore, it is necessary to use a filter to extract useful components and filter out the useless components and noise. Voltage-controlled voltage source model and double selection of second order ring are two types of filter, including voltage-controlled voltage source model has the advantages of low components number, low output impedance, can obtain higher accuracy of numerical and gain, easy to adjust, the characteristics of high sensitivity; Dual quadratic ring has a number of components, stable performance, convenient adjustment, low sensitivity, and high performance many integrated active filter, active filter and also mostly with double second order loop circuit as the prototype.

In order to be able to use digital circuits to process analog signals, analog signals must be converted to corresponding digital signals through $\mathrm{A} / \mathrm{D}$ converters. $\mathrm{A} / \mathrm{D}$ converter is an important link in the forward channel of the data acquisition system, which affects the subsequent signal processing time and the demodulation accuracy of the measured signal. In a data acquisition system, the sampling speed represents the realtime performance of the acquisition system. The sampling speed is determined by the analog signal bandwidth, the number of data channels and the number of samples per cycle. For high speed circuits, A/D can be used to meet the system requirements by using successive approximation, high resolution, high conversion rate, and A chip with sampleholding device.

In addition, according to system requirement and configuration, reasonable design of system power supply, selection of DSP, FPGA device, depending on the actual design meets the requirements of software process and display, control, alarm and other related content.

\section{CONCLUSION}

According to the power system when large current technical features, technical requirements, test standard requirements, system research of the full optical fiber current sensor principle of technical characteristics, composition, working principle and so on. On the basis of the breakthrough of the full optical fiber current sensor technology bottleneck, developed the high measurement precision, high stability, high freezing, with independent intellectual property rights, independent development ability of large current full optical fiber detection system, for its application in electric power industry to lay a solid foundation.

\section{ACKNOWLEDGMENT}

Thanks to the collaborators for the data support of this paper, thanks for the support of project funds.

\section{REFERENCES}

[1] The Nonreciprocal Errors in Fiber Optic Current Sensors[J]. Wei Wang,Xuefeng Wang,Junlei Xia. Optics and Laser Technology. 2011 (8)

[2] Improving optical fiber current sensor accuracy using artificial neural networks to compensate temperature and minor NOn-Ideal effects. Antonio C Zimmermann,Marcio Besen,Leonardo S Encinas,et al. Proceedings of SPIE the International Society for Optical Engineering. 2011

[3] Fiber optic current sensor based on special spun highly birefringent fiber Nai Peng,Yong Huang,Shuangbao Wang,et al. IEEE Photonics Technology Letters. 2013

[4] Thermooptically induced bias drift in fiber optical Sagnac interferometers. Mohr,Friedemann. Journal of Lightwave Technology. 1996 